\title{
Acuerdo de paz y crecimiento económico
}

\author{
Juan Héctor Vidal \\ Miembro del Senado Consultivo UTEC
}

\section{NOTA INTRODUCTORIA}

El Acuerdo de Paz firmado en Chapultepec el 16 de enero de 1992, constituye sin duda uno de los hechos más importantes desde que El Salvador surgió a la vida independiente. ES más, en la historia reciente, ese acontecimiento constituye un hito a nivel latinoamericano, porque significó ponerle fin a un largo conflicto interno por la vía negociada, lo que trajo consigo, entre otras cosas, algo inédito: la transformación de una de las fuerzas contendientes en partido político.

Habiendo coincidido la guerra civil con una etapa en la que toda América Latina atravesaba por grandes problemas económicos, cuya duración y profundidad dieron lugar a la llamada "década perdida" -y que en el caso salvadoreño fue más aguda precisamente por los efectos directos del mismo conflicto- se esperaba que con su finalización, el país retornara a la normalidad económica. Además, el modelo económico que se había puesto en marcha años antes -sustentado en los principios de la economía de mercado, conforme a las ideas en boga- ya estaba generando resultados en el momento en que se firmó la paz.

En este escenario, todo parecía favorable para que el país se encaminara por nuevos patrones de crecimiento con mayor equidad social y donde el pluralismo ideológico actuaría como complemento ideal para consolidar la democracia. Obviamente, esta visión optimista no tomaba en cuenta la turbulencia que habría de caracterizar al mundo en los años venideros, como producto de eventos econó- micos sumamente complejos gestados en el exterior, donde con frecuencia se han mezclado con factores políticos, sociales, ambientales y hasta culturales. Aun así, repasar el comportamiento de la economía en los últimos veinte años -aunque sea de manera general- siempre resulta un ejercicio interesante.

Es en este marco, que la Secretaria de Cultura -al cumplirse un aniversario más del Acuerdo de Paz- organizó una serie de eventos dentro los que se incluyó un conversatorio entre economistas nacionales, para tratar de discernir sobre lo acontecido en el campo económico durante las últimas dos décadas.

Lo que sigue, se basa en la participación del autor en dicha actividad y la cual ha sido adaptada para la Revista Entorno de la Universidad Tecnológica de El Salvador, con las limitaciones que impone el tipo de evento que sirvió de escenario para el intercambio de opiniones.

\section{UN INTENTO DE INTERPRETACIÓN}

Se puede decir con alguna propiedad, que el tema económico ocupa un lugar relativamente limitado en el Acuerdo de Paz. Las explicaciones pueden ser variadas, pero ninguna de ellas, por sí sola, alcanza el nivel de contundencia cuando en retrospectiva, se valora el significado de la economía en el marco de un evento que habría de cambiar muchos aspectos de la vida nacional.

10 
Versiones de personas vinculadas con la negociación, indican que este aspecto solo fue abordado en las últimas jornadas del proceso, no porque se ignorara su importancia, sino porque el tiempo apremiaba y el énfasis estaba puesto mayormente en aquellos temas más directamente relacionados con las condiciones para el cese del fuego y el fortalecimiento de la institucionalidad democrática. Esto puede ser una explicación razonable y entendible.

Sin embargo, el que el FMLN no haya hecho un asunto de honor ni siquiera sobre la forma en que se estaba conduciendo la política económica, pudo haber respondido a dos razones: primera, a que ya en 1991 el aparato productivo estaba dando muestras claras de recuperación, con base a un modelo que privilegiaba el mercado. Segunda, a que con el derrumbamiento del socialismo real, la izquierda de todo el mundo, había visto debilitado su esquema ideológico sobre el papel del papel del Estado en la economía.

Bajo estas circunstancias, el gobierno, a través de sus negociadores, podía también darse el lujo de mirar hasta con desdén cualquier intento de la contraparte de atacar un esquema de gestión económica que estaba siendo exitoso, que obviamente contaba con el beneplácito de la empresa privada y en general de la población, por los beneficios que estaba generando en términos de empleo y una mayor disponibilidad de bienes y servicios a precios más accesibles, en comparación con las condiciones prevalecientes en una economía de guerra.

Sánchez Cerén, en sus memorias pone el tema en los siguientes términos: "De hecho, nunca (el FMLN) ha tratado de incidir para que la política económica del país diera respuesta a los problemas que originaron la guerra (...) Firmamos los acuerdos en un escenario neoliberal que el gobierno arenero siguió practicando a sabiendas de que esa política avalada por el Banco Interamericano de Desarrollo, el Banco Mundial y el Fondo Monetario Internacional, era contraria a los Acuerdos de Paz, que exigían un Estado Social redistributivo"1

Puede decirse entonces que el FMLN se limitó prácticamente a tomar como un dato el curso que llevaba la política económica hasta ese momento. Esto lo confirma el

1 Sánchez Cerén, Salvador: Con Sueños se escribe la historia", pág. 240 preámbulo al tema económico y social del Acuerdo, que en la parte pertinente dice lo siguiente:

"Además, si bien la filosofía u orientación general de la política económica del gobierno, que el Frente no necesariamente comparte, no son parte de este Acuerdo, ambas partes coinciden en la necesidad de ofrecer algunas orientaciones básicas que permitan generar la estabilidad social necesaria en el periodo de transición, consolidar la paz y avanzar hacia la reunificación de la sociedad salvadoreña"

Siendo puristas, no se percibe pues, ni siquiera un atisbo de repudio al modelo económico que estaba en marcha, aunque para lograr esas orientaciones básicas, se creó el Foro para la Concertación Económica y social. A esta instancia tripartita (Gobierno, sector privado y sindicatos), se le encomendó la misión de lograr "un conjunto de amplios acuerdos, tendentes al desarrollo económico y social del país, en beneficio de sus habitantes".

Sin duda, estos propósitos estaban revestidos de mucha racionalidad, pero en los hechos, no formaban parte, ni remotamente, de la agenda que se había trazado la administración Cristiani. Tan es así, que dicha instancia desapareció en poco tiempo, porque en un ambiente de poco apoyo político, no se encontró la forma de conciliar los objetivos del Foro, con la agenda económica del gobierno que en todo caso, estaba enmarcada en los programas de estabilización y ajuste que patrocinaban el Banco Mundial y el Fondo Monetario Internacional como lo estaba haciendo la mayoría de los países latinoamericanos, para hacer frente a los grandes desequilibrios reales y financieros asociados con la llamada crisis de deuda.

Personalmente tuve la oportunidad de vivir la experiencia del Foro y en retrospectiva, tiendo a coincidir en que con su desaparición, se perdió una valiosa oportunidad de concertar iniciativas y acciones para impulsar el proceso de desarrollo económico, bajo patrones que se hicieran cargo de aquellos problemas que en el pasado habían obstaculizado el logro de mejores condiciones de vida para grandes segmentos de la población y que algunos consideran que alguna influencia tuvieron en el desencadenamiento del conflicto armado. 


\section{ECONOMÍA Y CULTURA DE PAZ}

Más allá de cualquier fundamento ético que pudiera estar presente en el programa económico del gobierno de turno, lo cierto es que desde de 1990 había empezado a registrarse un cambio de tendencia importante en la economía, después de un largo período de aletargamiento, con retrocesos importantes en algunos años, coincidiendo precisamente con el inicio del conflicto armado y con el ciclo depresivo que vivió toda América Latina.

El hecho de que el gobierno compartiera con los empresarios los principios de la economía de mercado, era un factor determinante para estimular la inversión y el empleo, que circunstancialmente son hoy en día, motivo de mucha preocupación ante el prolongado y lento crecimiento de la economía, hoy en día agravado por el impacto de la crisis internacional, principalmente en la eurozona.

A partir de los primeros logros en el plano económico y por una lógica elemental, se podía suponer que con el advenimiento de la paz, el país estaría en mejores condiciones para sustentar un crecimiento robusto y sostenido en el largo plazo. Aun aceptando esa hipótesis, resultaba riesgoso asumir la tesis simplista de que bastaba con silenciar las armas y el mero cambio en la gestión económica, para que el sistema (económico) empezara a reconstruirse, haciéndose cargo, de manera automática, de las inequidades que aquél supuestamente generaba. Pero en los hechos, al menos en materia de crecimiento, la economía se desempeñó muy positivamente durante toda la primera mitad de los noventas.

Sin embargo, esto no era suficiente. Si la economía había comenzado a crecer vigorosamente, lo ideal hubiera sido dedicar un mayor esfuerzo a la parte social. Esto significaba descartar la teoría del rebalse, para conciliar el objetivo de la eficiencia económica, con la necesidad de canalizar responsablemente más recursos públicos hacia los sectores más vulnerables. De hecho, esto se perfilaba como la única vía para que el país pudiera rescatar lo perdido y empezar a actuar más en consonancia con el espíritu de Chapultepec. Guardar ese equilibrio resultó virtualmente imposible, pues ya sobre la marcha, el programa económico de la administración Cristiani se fue nutriendo cada vez mas de la corriente reformista que se extendió a lo largo y ancho de
América Latina, con un enfoque muy economicista, Esto, bajo el supuesto que el mero crecimiento crearía automáticamente más empleo y este a su vez incidiría positivamente en la reducción de la pobreza. ${ }^{2}$ En esto hay que resaltar el hecho de que los programas de ajuste y estabilización, importantes como pudieron ser para el ordenamiento de la economía y en el asentamiento de bases para el crecimiento sostenido, no se hacían cargo -ni tenían por qué haberlo hecho- de especificidades, como las asociadas directamente al conflicto armado.

Además, el hecho que El Salvador dependiera excesivamente de la cooperación de los Estados Unidos en materia económica y militar, presionaba en gran medida para que el país se sometiera a los dictados de los organismos internacionales, postulados que después encontraran el sello político en el llamado Consenso de Washington, donde la economía debía guiarse, en palabras del presidente Reagan, por la "magia del mercado".

Atrás fueron quedando los planteamientos de FUSADES que allá por 1987 lanzó sus primeras ideas sobre la necesidad de un nuevo modelo económico, basado en la experiencia de los países del sudeste asiático y en el modelo de la economía social de mercado que adoptó Alemania después de la Segunda Guerra Mundial. Los planteamientos de dicha institución -que sirvieron de base para la formulación del programa económico y social del primer gobierno de ARENA- sin ser conflictivos en su esencia con los que en un principio guiaron la gestión de Cristiani, se mediatizaron a partir del enfoque cada vez más "eficientista", como se sugirió antes, que le imprimieron los responsables de la gestión económica.

Tan fuerte llegó a ser la adhesión a ese enfoque, que en su momento se empezó a escuchar la versión de que un tema obligado en las tertulias de sobremesa de los funcionarios del Banco Mundial y del FMI, era la capacidad demostrada por El Salvador para poner en práctica sus recomendaciones. En cualquier caso, esto encajaba perfectamente

2 Vidal, Juan Héctor. "El papel del sector privado en la reducción de la pobreza. Ponencia presentada en la Conferencia Internacional: Reducción de la Pobreza en América Latina y el Caribe: retos para el Nuevo Milenio, Toronto, Canadá 1996. En Vidal, “Por los senderos de la transición". Universidad Tecnológica de El Salvador, 1997, pág. 226

12 
en el nuevo orden mundial que se estaba perfilando con el fenómeno de la globalización y las teorías en boga particularmente sobre cómo lidiar con los desajustes reales y financieros en los países en proceso de desarrollo. ${ }^{3}$ En retrospectiva, se puede decir que fue el giro que dio eventualmente la gestión económica bajo el gobierno de Cristiani, lo que dificultó que en paralelo a la cultura de paz, se impulsara la estructuración de un sistema productivo más eficiente, pero con mayor contenido social.

A partir de estos acontecimientos, la reforma económica siguió su curso, donde los principales espacios fueron ocupados por la indiscriminada y rápida apertura de la economía, una reforma tributaria claramente sustentada en los gravámenes indirectos, la desregulación de los mercados y, por supuesto, por la privatización del sistema bancario y la desnacionalización del comercio exterior.

En ese marco, la economía continuó creciendo a un ritmo acelerado durante todo el primer quinquenio de los noventas, al punto de ubicarse en el segundo lugar en el contexto latinoamericano, después de Chile. (Cuadro $N^{\circ} 1$ )
Esa tendencia se extendió hasta 1995, ya bajo la Administración Calderón Sol; pero a partir de 1996, el sistema cayó nuevamente en un aletargamiento que se agudizó en el 2001, coincidiendo con la peor decisión que se le atribuye a los cuatro gobiernos de ARENA: la dolarización de la economía y los efectos destructivos de los sismos en enero y febrero de este último año.

Este quiebre, además de inesperado, fue muy pronunciado. En efecto, después de haber crecido durante los cinco años previos a una tasa promedio superior al 6\%, el PIB sólo aumentó $1.7 \%$ en 1996, para cerrar el segundo quinquenio de la década con una expansión media apenas superior al 3.0\%. Este comportamiento llevó al país a rezagarse económicamente respecto al resto de América Latina, pasando del segundo lugar en el primer quinquenio al décimo puesto en el período. (Cuadro $\mathrm{N}^{\circ}$ 2)

Esto último se dio, a pesar del optimismo que por esa época despertaba la privatización. Como se sabe en la agenda principal del proceso estaban las telecomunicaciones, las distribuidoras de energía eléctrica y el sistema previsional.

CUADRO N $\mathrm{N}^{\circ} 1$

Tasas de crecimiento promedio del PIB en América Latina (1991/1995)

\begin{tabular}{|c|c|c|}
\hline Ranking & País & Tasa \\
\hline 1 & Chile & 8.7 \\
2 & El Salvador & 6.2 \\
3 & Argentina & 5.8 \\
4 & Perú & 5.7 \\
5 & Panamá & 5.5 \\
6 & Costa Rica & 5.5 \\
7 & Rep. Dominicana & 5.3 \\
8 & Colombia & 4.6 \\
9 & Guatemala & 4.3 \\
10 & Bolivia & 4.1 \\
\hline
\end{tabular}

\begin{tabular}{|c|c|c|}
\hline Ranking & País & Tasa \\
\hline 11 & Uruguay & 4.0 \\
12 & Paraguay & 3.8 \\
13 & Honduras & 3.6 \\
14 & Venezuela & 3.5 \\
15 & Brasil & 3.1 \\
16 & Ecuador & 2.7 \\
17 & Nicaragua & 1.8 \\
18 & México & 1.6 \\
19 & Haití & -2.2 \\
20 & Cuba & -6.8 \\
\hline
\end{tabular}

Fuente: CEPAL.

3 Según Stigliz, en lo que concierne a la austeridad fiscal, la privatización y la liberalización de los mercados sobre todo la apertura comercial; las políticas relacionadas fueron diseñadas para responder a problemas muy reales de América Latina, aceptando que, por esa razón, tenían mucho sentido. (Citado por Juan Héctor Vidal, en “De la Ilusión al Desencanto...... pág.. 50)
Todo esto tuvo lugar a partir de 1998, pero aparte del importante flujo de capital extranjero implicado, el impacto no se vio reflejado en el comportamiento de la economía, aunque sí se fortalecieron las finanzas públicas, que se habían 
Cuadro $\mathrm{N}^{\circ} 2$

TASAS DE CRECIMIENTO PROMEDIO EN AMÉRICA LATINA

$(1995 / 2000)$

\begin{tabular}{|c|c|c|c|c|c|}
\hline Ranking & País & Tasa & Ranking & País & Tasa \\
\hline 1 & República Dominicana & 6.7 & 12 & México & 3.5 \\
\hline 2 & Chile & 5.3 & 13 & Honduras & 3.2 \\
\hline 3 & Nicaragua & 5.2 & 14 & América Latina & 2.7 \\
\hline 4 & Costa Rica & 4.8 & 15 & Brasil & 2.4 \\
\hline 5 & Cuba & 4.2 & 16 & Argentina & 1.7 \\
\hline 6 & Panamá & 4.2 & 17 & Colombia & 1.7 \\
\hline 7 & Guatemala & 4.1 & 18 & Uruguay & 1.6 \\
\hline 8 & Haití & 3.8 & 19 & Venezuela & 1.4 \\
\hline 9 & Bolivia & 3.7 & 20 & Ecuador & 1.1 \\
\hline 10 & El Salvador & 3.6 & 21 & Paraguay & 0.8 \\
\hline 11 & Perú & 3.5 & & & \\
\hline
\end{tabular}

Fuente: CEPAL

venido debilitando, como producto de los compromisos adquiridos con el Acuerdo de Paz. En cambio, el proceso como tal fue cuestionado, por la falta de transparencia, al menos el caso de los servicios estratégicos mencionados.

Situándonos en una realidad más cercana, podemos traer a colación el estudio realizado para justificar la concreción de la iniciativa "Asocio para el Crecimiento", que es un proyecto de la administración obama que incluye sólo cuatro países a nivel mundial. Éste sostiene que la economía del país sólo creció a un promedio anual del 2\% de 2000 al 2010, ubicándose muy por debajo de la media latinoamericana. En la gráfica siguiente se muestra el desempeño de la economía salvadoreña frente al resto de Centroamérica en este período.

Más allá de las repercusiones que han tenido los fenómenos naturales en el pobre desempeño de la economía, es claro que éste es un fiel reflejo de la presencia de problemas estructurales no resueltos, donde sobresalen la disfuncionalidad de la relación ahorro-inversión, el desajuste fundamental en las cuentas externas y más recientemente, el desequilibrio fiscal.

Ese macro desempeño se expresa muy claramente en el hecho de que la economía salvadoreña ha estado a la zaga en el contexto latinoamericano y en el último lugar en el ámbito centroamericano. En este último caso, es importante resaltar el hecho de que la producción de bienes y servicios cada vez más pierde importancia en la generación del PIB regional, descendiendo de 22\% en 1995 a un estimado del 18\% en 2010 según cifras del Consejo Monetario Centroamericano. Este no es un asunto trivial, si se considera que El Salvador fue el país de la región que más profundizó en la reforma económica.

Gráfico No. 1

Centroamérica: Crecimiento del PIB

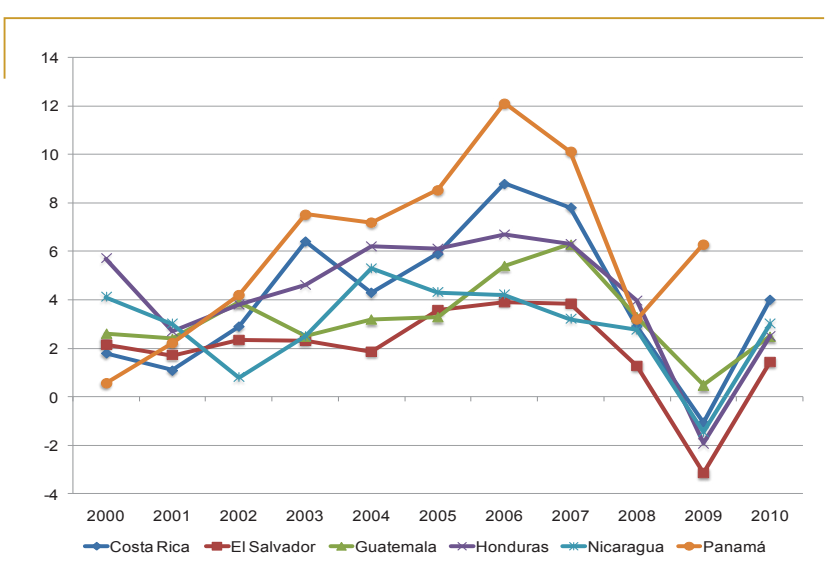

Fuente: CEPAL

14 
La inversión extranjera, que en cualquier país es un soporte fundamental para sustentar altas tasas de crecimiento en el tiempo, registra asimismo flujos excesivamente bajos, lo que nos coloca también en este plano, en lugares similares a los que ocupamos en materia de crecimiento. Solo en aquellos años que coinciden con la privatización de los sectores estratégicos y con la venta de los bancos a grandes conglomerados extranjeros, se observa un sensible aumento en esos flujos. En este caso, los otros países centroamericanos indudablemente ofrecen mejores condiciones que el nuestro.
Aparte del lento crecimiento, arrastramos un gran desequilibrio con el exterior que solo es posible enfrentarlo con el producto de las remesas. Por otro lado, cada vez se presenta más crítica la situación fiscal, único campo de acción que nos quedó para hacer política económica después de la dolarización. Solo el tamaño de la deuda pública es un enorme obstáculo para mantener unas finanzas públicas sanas y el mismo régimen monetario, pues ya supera el $50 \%$ del PIB. Con esto se dificulta más retomar la senda del crecimiento y enfrentar con mayor eficacia los marcados déficits que venimos arrastrando en el campo social. Con estas restric-

Cuadro $\mathrm{N}^{\circ} 3$

Centroamérica: Inversión Extranjera Directa

(Millones US\$)

\begin{tabular}{|l|c|c|c|c|c|}
\hline \multicolumn{1}{|c|}{ Año } & $2000-2005$ & 2007 & 2008 & 2009 & 2010 \\
\hline Costa Rica & 597 & 1,896 & 2,021 & 1,323 & 1,412 \\
\hline El Salvador & 325 & 1,508 & 784 & 431 & 89 \\
\hline Guatemala & 334 & 745 & 754 & 574 & 678 \\
\hline Honduras & 418 & 928 & 1,006 & 523 & 798 \\
\hline Nicaragua & 219 & 382 & 626 & 434 & 508 \\
\hline Panamá & 656 & 1,777 & 2,402 & 1,773 & 2,363 \\
\hline \multicolumn{1}{|c|}{ Total } & 2,549 & 5,728 & 7,593 & 5,058 & 5,845 \\
\hline
\end{tabular}

Fuente: CEPAL

Siendo objetivos, podemos decir que el comportamiento de la inversión extranjera refleja con nitidez lo poco atractivo que resulta un país, que si bien ha dado pasos importantes en la consolidación de la democracia y en la liberalización y ordenamiento de su economía, arrastra grandes problemas en materia de infraestructura, recursos humanos, transparencia, delincuencia y, de manera muy importante, en seguridad jurídica.

Al margen de las falencias reales e institucionales que confrontamos, la inversión extranjera y por supuesto la nacional, han sido afectadas por el discurso recurrente de la oposición más radical, que con frecuencia envía mensajes parecidos a los del presidente venezolano.

Si nos fuéramos a guiar por los presupuestos básicos del Acuerdo de Paz, todo esto debería de ser un poco diferente. En efecto, contrario a lo que se visualizaba en 1992, cuando el sistema productivo nacional estaba dando muestras claras de dinamismo, la economía atraviesa actualmente por serios problemas. ciones fiscales, se hace hasta más difícil enfrentar con alguna posibilidad de éxito la delincuencia desbordada.

En cambio, lo que ha venido sosteniendo la economía, es el consumo desmedido que se financia en gran parte con el producto de las remesas, para desembocar en una especie de círculo vicioso difícil de romper, simplemente porque no se sabe por dónde comenzar o no se tiene la voluntad ni el espacio social, para someter a la economía al ajuste que se requiere en estos casos.

La siguiente gráfica muestra cómo el PIB, representado por la línea horizontal ha sido superado desde 1999 por el consumo agregado. Este fenómeno constituye en la práctica, uno de los desequilibrios estructurales más graves de la economía, signo inequívoco de que esta se ha tornado totalmente improductiva y dependiente del ahorro externo. 
GRAFICA N ${ }^{\circ} 2$

El Salvador: una economía basada en el consumo

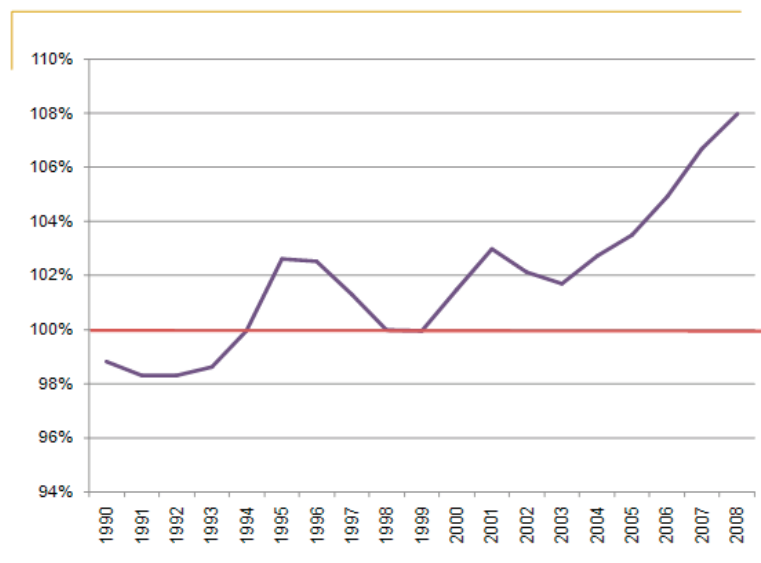

Fuente: PNUD

Una síntesis del comportamiento de la economía en las últimas décadas, se presenta en el cuadro siguiente, que incorpora los indicadores económicos más relevantes desde 1990 primer gobierno de ARENA hasta 2011.

\section{REFLEXIONES FINALES}

A partir del escenario planteado, se puede concluir que veinte años después, existe en el plano económico una brecha considerable entre aspiraciones y realizaciones. Solo con poner la atención en los lugares que ocupamos en el contexto latinoamericano e incluso en el ámbito regional en materia de crecimiento económico, ya se puede percibir lo poco exitosos que hemos sido en construir un sistema productivo eficiente, con bases solidas de sustentación en el tiempo.

Esto no ignora el contexto internacional que nos ha afectado, incluso mucho más que los países vecinos, a partir de decisiones cuestionables como la dolarización y la presencia de otros factores, como la inseguridad jurídica, la delincuencia desbordada y, sin duda, el futuro político.

En este último plano, si bien les llevamos una gran ventaja a países como Nicaragua, Venezuela, Ecuador y Bolivia, donde los gobiernos se han entronizado en el poder prostituyendo las instituciones, no hemos sido capaces de caminar unidos por la senda que nos trazamos en Chapultepec. El riesgo de seguir la senda de esos países, siempre estará latente, mientras la izquierda más radical siga pensando en que la opción es el socialismo del siglo XXI.

Y en alusión más directa al tema estrictamente económico, creo que no hemos sido capaces de aprovechar nuestra energía creadora. De hecho, nos ha faltado coraje para hacer los cambios que eran necesarios para construir un sistema eficiente que distribuyera más eficientemente el ingreso, sin intervencionismos estatales odiosos.

Estamos claros que esto requería un esfuerzo denodado y una visión compartida, para hacer del tema económico, la plataforma que nos catapultaría hacia nuevos estadios de desarrollo, en su más amplia expresión. Obviamente,

CUADRO N ${ }^{\circ} 4$

Evolución de variables claves 1990-2011 (promedios anuales)

\begin{tabular}{|c|c|c|c|c|}
\hline Período & $\begin{array}{c}\text { Crecimiento PIB } \\
\text { (promedio) }\end{array}$ & $\begin{array}{c}\text { Inflación } \\
\text { (promedio) }\end{array}$ & $\begin{array}{c}\text { Déficit } \\
\text { comercial \% PIB }\end{array}$ & $\begin{array}{c}\text { déficit Fiscal*\% } \\
\text { PIB }\end{array}$ \\
\hline $1990-1994$ & $5.9 \%$ & $13.9 \%$ & $15.9 \%$ & $2.0 \%$ \\
\hline $1995-1999$ & $3.9 \%$ & $4.8 \%$ & $13.8 \%$ & $2.0 \%$ \\
\hline $2000-2004$ & $2.1 \%$ & $3.3 \%$ & $16.6 \%$ & $3.6 \%$ \\
\hline $2005-2009^{\mathrm{a}}$ & $2.2 \%$ & $3.7 \%$ & $21.8 \% \%^{\mathrm{b}}$ & $3.3 \%$ \\
\hline $2010-2011$ & $1.5 \%$ & $3.6 \%$ & $20.5 \%$ & $4.2 \%$ \\
\hline
\end{tabular}

*Déficit fiscal incluye pensiones

a/ Con datos de 2009 a partir de estimaciones oficiales al 21 de diciembre de 2009.

b/ Promedio período 2005-2008.

Fuente: Elaboración propia con datos del BCR y Ministerio de Hacienda

16 
hicimos las reformas siguiendo el paradigma que nos plantearon los organismos internacionales; el problema radica en que en temas como la privatización, la sanidad fiscal, la apertura comercial y la desregulación, hemos cometido grandes errores por acción u omisión.

En la práctica, progresivamente fuimos desarticulando el tejido productivo bajo la prédica de que lo sabio era importar e importar para poder exportar. Como un subproducto no muy halagüeño, hoy nos distinguimos por ser uno de los mayores consumistas en el mundo, respecto al tamaño de la economía,

Y todo esto ocurría, en circunstancias en que la clase media se debilitaba, estrato que en cualquier país constituye un elemento central para que la democracia funcione a plenitud. En cambio, hicimos de la emigración el punto de escape para una situación social que no pudo ser alterada significativamente ni con el advenimiento de la paz. Como corolario, sustituimos el esfuerzo genuino en torno a las tareas del desarrollo económico y social, confiados en que las remesas familiares harían la diferencia.

La euforia que ha creado la nueva extensión del TPS, es la más clara expresión de que nos hemos acomodado a una situación que, en general, no se condice con el espíritu del Acuerdo de Paz, ni en lo económico, ni en lo social. Y menos con aquella credencial que tuvimos antes del conflicto, de ser un país emprendedor por excelencia.

De cara al futuro, hay mucho escepticismo sobre nuestra voluntad para cambiar el status quo. Al menos en estos momentos, el contexto internacional no nos favorece en nada y más bien, nos expone ante una mayor vulnerabilidad en comparación con países de similar estado de desarrollo relativo, como los vecinos centroamericanos.

Hay por supuesto iniciativas en marcha, como las que se están impulsando con la cooperación de los Estados Unidos (Socios para el Crecimiento y un segundo FOMILENIO), que pueden de alguna manera ayudarnos a sortear las dificultades que estamos viviendo y las que se perfilan en el horizonte. El problema en este plano, radica en las diferencias marcadas existentes entre el gobierno y el sector productivo nacional

Y en el campo político, si bien existe una marcada incertidumbre, coincido con aquellos que todavía estamos a tiempo de plantearnos la clase de sociedad que queremos heredarles a las futuras generaciones. La última edición de ENADE, es en este sentido prometedora, siempre que la clase política entienda que para enrumbar al país por un nuevo derrotero, es indispensable trabajar a partir de una visión compartida.

En esta misma línea, cada vez se está extendiendo más la idea de que el país necesita un nuevo acuerdo de paz. Este mero hecho insinúa que muchos salvadoreños estamos genuinamente decididos a enfrentar con coraje la situación general imperante.

Ciertamente 20 años pueden ser muy pocos para materializar un sueño como aquel que compartimos como sociedad el 16 de enero de 1992. Lo importante es estar conscientes de que sólo progresaremos como país, si hacemos un esfuerzo genuino para unir esfuerzos y voluntades para construir una sociedad cualitativamente diferente. 